\title{
La salud pública en el mundo actual.
}

\section{World's public health today.}

\section{SOTELO, J. Manuel ${ }^{1}$}

${ }^{1}$ Representante de las Organizaciones Mundial y Panamericana de la Salud en México. Trabajo presentado en la Facultad de Medicina de la Universidad Nacional Mayor de San Marcos.

\section{INTRODUCCIÓN}

La meta social de Salud para todos, adoptada por los gobiernos del mundo representa un conjunto de valores fundamentales para contribuir al desarrollo. Las apreciaciones a las que me voy a referir les ruego las tomen en relación al convencimiento de que la salud puede contribuir al desarrollo, es parte de él y se identifica con el bienestar.

La nueva década de los noventa se inaugura con un panorama reordenador, que abarca todos los ámbitos del desarrollo humano. Así, en lo político, se viene suscitando todo un proceso democratizante, que rompe con viejas estructuras de poder y da cabida a esquemas participativos; en lo económico, se registran drásticos cambios, que redefinen los papeles del Estado y el mercado y establecen las bases de una integración mundial; y en lo social, aunque se continúa con graves diferentes entre individuos, países y regiones, se observan progresos significativos en varios indicadores, sustentados en un amplio consenso internacional orientado a la justicia y equidad.

Inmersa en este gran marco, la salud como medio y fin del desarrollo, tiene que delinear su espacio a partir de profundas transformaciones en sus sistemas y programas, que tienden a acompañar la gradual y diferenciada transición epidemiológica que está sucediendo en las diferentes realidades nacionales y que en muchos obedece a las diversas formas de participación, producción y consumo, así como a los rezagos que las diferentes economías presentan.

Teniendo en cuenta este complejo y cambiante escenario, se pretende hacer una breve revisión de la situación mundial, caracterizando en términos generales las macrotendencias observables; definir en ese marco el fenómeno de transición epidemiológica, mediante la relación de algunos factores que favorecen su estado actual en realidades específicas; identificar algunas importantes iniciativas de cambio que se operan en el sector salud; analizar la aplicación de estas modificaciones en las diferentes regiones o países; y por último, hacer alguna reflexión sobre el significado y dimensión que va tomando la salud pública ante las nuevas perspectivas del desarrollo humano.

\section{ANÁLISIS DE LA SITUACIÓN MUNDIAL}


En la actualidad el desarrollo humano ha evolucionado a concebirse como el mejoramiento sostenible del nivel de vida, el cual comprende aspectos tales como la mayor oportunidad de igualdades, el consumo material, el derecho a la educación y la salud, la libertad política, las libertades civiles y la protección al medio ambiente. Dentro de este enfoque, primordialmente humanista, el objetivo global del desarrollo es dotar de mayores posibilidades y derechos en lo económico, político y civil a todas las personas, sin distinción de sexo, grupo étnico, religión, raza, región o país.

Asimismo, se tiene que tomar en cuenta que las prioridades de las personas no están determinadas para siempre y que éstas cambian con el tiempo, a medida que las circunstancias y las aspiraciones varían, todo lo cual debe considerarse en su conjunto para que los hombres, las mujeres y los niños sean el centro de atención, a partir del cual se forje el desarrollo.

Hay que tener en cuenta también que este desarrollo requiere del denominado "crecimiento económico", que por sí mismo no sería capaz de lograr un mejoramiento sostenido del bienestar humano, requiriéndose de una firme acción, basada en políticas sólidas tanto en lo económico, como en lo social.

Partiendo de este marco, se puede apreciar que en la actualidad se producen notables cambios en la economía y la política mundiales. Es así como más de una decena de países han aprendido reformas económicas fundamentales; la democracia se ha extendido por toda Europa Oriental y actualmente se abren grandes interrogantes sobre el futuro de los países integrantes de la que fue la Unión Soviética; se derrumbó en muro de Berlín; Alemania sufre un proceso de reunificación; y en toda África caen los sistemas unipartidistas. Sin embargo, antes este panorama alentador, existen regiones del mundo donde más de mil millones de personas, es decir, una $5^{\mathrm{a}}$ parte de la población del planeta, subsisten con menos de 1 dólar al día y otras que se han visto ampliamente lesionadas por la llamada "década pérdida de los 80s". Un acercamiento mayor a la unión política y económica de la Comunidad Económica Europea, una mayor concentración de la pobreza absoluta muy obstenible en África y la crisis de la deuda externa en América Latina, son expresiones de la situación actual.

La velocidad de los cambios en el mundo y su profundidad, revisten particular importancia, que compromete prácticamente todos los aspectos de la vida y sus vertientes políticas económicas, sociales y, naturalmente está teniendo y tendrá impactos sobre el quehacer en salud.

Destacan las siguientes situaciones globales:

- El nuevo orden mundial, con reorganización de la actividad productiva y del comercio, con realineamiento del ejercicio del poder y de la política internacional, representa una oportunidad y a la vez un riesgo, oportunidad para aumentar la capacidad de producción del mundo en su conjunto pero riesgo de relegación para los países subdesarrollados, especialmente para América Latina. 
- La presencia creciente de sentimientos nacionalistas y el renacimiento de los valores étnicos, que en alguna medida entran en conflicto con la tendencia de globalización, amenazan la paz y desvían la atención de oportunidades de cooperación.

- El crecimiento vertiginoso de la ciencia y la tecnología con inmensas posibilidades de intervención en salud, particularmente en la prevención y el control de enfermedades, con desarrollo de vacunas y drogas, facilitado por la ingeniería genética y la biotecnología.

- La tremenda disparidad entre ricos y pobres en las naciones y entre los grupos sociales en cada país. Esta situación la califica el Director de la OPS, el Dr. Guerra de Macedo, como la configuración del gran conflicto del futuro, la gran amenaza a la estabilidad de la humanidad.

- La visión neoliberal para el proceso de desarrollo integral, que podría significar riesgos para la acción en todos los ámbitos de la actividad social.

- La valoración del ambiente y las relaciones de las sociedades con él. El problema ambiental existe como uno de los grandes conflictos y temas con implicancias en la salud y su quehacer.

Por su parte América Latina, después de un período de elevado dinamismo que se sustentó en un modelo económico caracterizado por un Estado desarrollista, que se expandía y asumís diversas formas de generación de empleo, de promoción de servicios públicos y de apoyo a la empresa privada, alimentado por excedentes generados de los sectores tradicionales de exportación y, cuando éstos se agotaron, por un creciente financiamiento externo o en otros casos por financiamiento inflacionario, cayó para los años 80 en una aguda crisis económica que dio lugar a una recesión generalizada, caracterizada por un notable decrecimiento del PBI por habitante, que se redujo $8.3 \%$ en el decenio; un deterioro en los términos de intercambio, de $21 \%$; un aumento de la desocupación abierta y el desempleo, que para finales del decenio llegó a ser mayor que en 1980; una disminución de los gastos gubernamentales en los renglones sociales que ha potenciado las insuficiencias tradicionales de infraestructura básica y servicios públicos, incrementando notablemente la deuda social; la persistencia de la inflación, que pasó de 56\% en promedio en 1980 a 994\% en 1989; una cuantiosa transferencia de recursos al exterior, y la desigualdad y el empobrecimiento extremo crecientes, donde la población marginada pasó a representar un $40 \%$. Es justo reconocer que en la región, los indicadores sociales de resultado sostuvieron una mejoría, pero a un ritmo menor que la tendencia que se observó en las dos décadas anteriores. Como consecuencia de ello, el esfuerzo requerido en los noventa para lograr las metas sociales es ahora más alto, no sólo en las relacionadas con la salud y la educación, sino también con la dotación de otros servicios. En general, la deuda externa en los países en desarrollo creció un $1,000 \%$ en la época de crisis.

Ya para 1990, prácticamente se detuvo la expansión de la economía mundial. Se hizo patente la desaceleración de la actividad económica en varios de los países industrializados, acompañada de tasas crecientes de inflación, la cual se hizo más pronunciada con la crisis del Golfo Pérsico, en agosto de 1990.

Las necesidades financieras que plantearon la unificación de Alemania y la reconstrucción por causa de la guerra en el Medio Oriente, ejerciendo una presión 
alcista en las tasas de interés a corto plazo en Alemania y en Japón, pese a la desaceleración observada en 1990 y los primeros meses de 1991. En los países industrializados se redujo la tasa de crecimiento del PBI, de $4.5 \%$ en 1988 , a $2.6 \%$ en 1990. Cánada, Estados Unidos y el Reino Unido han sufrido recesión. El crecimiento económico también ha sido menor en los demás países de Europa Occidental.

En los países en desarrollo fue menor el crecimiento del PBI en términos reales, pasando de $4.3 \%$ en 1988 a $2.9 \%$ en 1989 y a $2.2 \%$ en 1990 , siendo éste el más bajo desde 1982. Las principales razones han sido la persistencia inestabilidad macroeconómica, las deficiencias de las políticas internas, la baja del precio de los productos básicos, con excepción del petróleo, las elevadas tasas de interés internacional y el crecimiento más lento del comercio exterior.

En América Latina, entre otras causas, por la gran recesión ocurrida en Brasil, se ha producido una acentuada contracción que va aparejada a la continuación de la crisis de la deuda. Sin embargo, existen signos alentadores re recuperación, a partir de nuevas reformas estructurales y el surgimiento de nuevos tratados comerciales entre países de la región y otras regiones del mundo. Dentro de lo último, tanto los tratados de libre comercio, (como el de México, Canadá y Estados Unidos; Argentina, Uruguay y Brasil; Colombia, México y Venezuela; países Centroamericanos y México; o el mercado común del Caribe), como la iniciativa para las Américas, presentada por el presidente de los Estados Unidos, ofrecen nuevas perspectivas en materia de comercio, inversión y deuda externa, así como a la paz social y al progreso y modernización de los sectores productivos.

En general, el mundo, ante la evolución seguida, se está viendo en la necesidad de otorgar un grado mayor de flexibilidad y agilidad a las relaciones económicas recíprocas entre países y regiones, en un marco de pluralismo político y democracia social participativa, en el cual el Estado debe asumir su papel regulador y dar cabida a la producción de bienes y servicios básicos para la población en general, de manera que se reduzcan las desigualdades sociales entre los grupos humanos, los países y las regiones. El estado necesario en este tipo de desarrollo, es un estado mucho más actuante, activo y presente.

Para ello, se están dando transformaciones político-administrativas en las naciones; los procesos productivos se organizan cada vez más en función de estrategias globales tendiendo a un sistema económico mundial; el proceso de globalización contrasta con la formación de regiones, como intento de regular la actividad económica, y la nueva revolución tecnológica está llamada a sufrir alteraciones profundas en bien de una mejor distribución.

Esta nueva orientación requeriría de una base de recurso humano saludable, bien formado y capaz de utilizar la tecnología más moderna, lo cual plantea como una prioridad fundamental del decenio de los noventa la inversión en capital humano, que eleve los niveles de alfabetismo e instrucción, estimulando el desarrollo profesional, científico y tecnológico. La creación de las condiciones para la producción y la productividad en un ambiente cada vez más complejo en el plano social, científico y 
tecnológico, exige la presencia de una institución social de las posibilidades del estado, y sobre todo, voluntad política que articule, que permita estabilidad y simultáneamente facilite un proceso de transformación sin quiebra de las pautas del marco de cada país.

Las privaciones en los países industrializados son de diferente dimensión y en ocasiones, naturales. Más de 1,000 millones de personas viven por debajo de la línea de pobreza, que en la Unión Soviética y Europa del Este, llegan a representar 200 millones; la tasa de desempleo oscila entre 6 y $10 \%$ en diez países y en otros tres supera el 10\%; los salarios de las mujeres son las dos terceras partes de los masculinos $\mathrm{y}$, en muchos de estos países, la estructura social sigue descomponiéndose rápidamente, registrándose altas tasas de drogadicción, suicidio, divorcio y familias monoparentales.

El indicador del Desarrollo Humano construido por el PNUD tomando en cuenta el ingreso, la esperanza de vida, el alfabetismo y los años de escolaridad, ha conducido a conclusiones interesantes. En la clasificación de los países industrializados, Japón se sitúa en primer lugar y Rumanía está en el último.

Entre los países en desarrollo, Barbados encabeza la clasificación y Sierra Leona ocupa el último lugar. Se destaca en esta lista la situación de Argentina, Venezuela y México del 43 al 45. Brasil, Colombia y Cuba ocupan los lugares 60, 61 y 62; Ecuador y Perú están en el 77 y 78, y Honduras y Bolivia junto con Haití en los lugares 100, 110 y 125. La lista que llega hasta el lugar 160 registra en los últimos lugares a países del Asia Oriental y Meridional, así como a otros del África.

En la clasificación según el índice de desarrollo humano, (IDH), 26 países se encuentran 20 o más puestos por debajo de lo que les correspondería según su ingreso per cápita, lo cual significa que podrían mejorar considerablemente sus niveles de desarrollo humano, si utilizaran más racionalmente sus ingresos nacionales.

En el ajuste por sexos, se muestra que existen graves disparidades en la mayoría de los países en desarrollo. Prueba de ello es que en Kenia el IDH femenino es apenas la mitad del masculino.

En los países industrializados las disparidades se han reducido, pero continúan siendo notorias en la educación terciaria, en la participación en la fuerza laboral y en las escalas salariales. Por ejemplo, en Japón la participación de las mujeres en el ingreso nacional es sólo el $26 \%$ de la de los hombres.

Siendo la distribución del ingreso otro factor de ajuste, se prueba que los logros del desarrollo humano pierden gran parte de su valor cuando no se comparten equitativamente. Así, cuando se pone en juego este valor, los IDH se reducen en más de $10 \%$ en varios países, incluidos Nepal y Brasil.

La libertad, que también ha servido de variable para ese tipo de determinaciones, contribuye a definir que los altos niveles de desarrollo humano tienden a lograrse en el marco de elevados niveles de libertad. 
Por último, la asignación de los gastos sociales en el sector público y de los provenientes de la ayuda internacional, demuestran que "la ausencia de compromiso político, y no la falta de recursos financieros, es con frecuencia la causa verdadera del abandono en que se encuentra el hombre". Ejemplo de ello son los gastos militares, el servicio de la deuda o las empresas públicas con pérdidas.

Se estima que la razón de gastos en proyectos de desarrollo humano tendría que ser de por lo menos un 5\% si un país desea mejorar sus condiciones. No obstante, en países como Brasil, Tailandia y Sierra Leona, el gasto gubernamental es muy elevado, 25 a $35 \%$ del ingreso nacional, pero menos del $2 \%$ se asigna a proyectos de prioridad humana.

A semejanza de lo que pasa a nivel nacional, los programas de ayuda internacional ofrecen un gran potencial cuya retribución podría ser enorme si sólo una $3^{\mathrm{a}}$. Parte de esos ingresos se destinará a áreas de prioridad humana, la que podría incrementarse cuatro veces.

\section{EL PANORAMA EPIDEMIOLÓGICO}

Si observamos los indicadores básicos de salud en los países del globo concluiremos, sin mayores análisis de asociación estadística, que son coincidentes, como ya se anotó con la polarización de los niveles de ingreso y la capacidad productiva. Sin embargo en algunos países estos indicadores no se correlacionan con su capacidad productiva ni, necesariamente, con la disponibilidad de calorías por habitante que, en cierto modo, es una expresión de la producción nacional de alimentos de la capacidad de compra.

La esperanza de vida se observa en sus niveles más altos en los países con latitudes más allá de los trópicos, lo mismo que las menores tasas de mortalidad infantil. También en estos países, especialmente del Hemisferio Norte, la disponibilidad de calorías por habitante se encuentra muy por arriba de las 3,000 y aunque en algunos países del espacio intertropical la disponibilidad de calorías per cápita es teóricamente suficiente (México por ejemplo), su origen es predominante de cereales y otros vegetales y, en menor escala, de proteínas de origen animal.

Llama la atención que en los tres países, que hasta 1988 registraban las menores tasas de mortalidad infantil, el ingreso de calorías per cápita no llegaba a una cifra de 3,000: Japón con la menor tasa de mortalidad infantil en el mundo, de 5 x 1,000 nacidos vivos registrados, Taiwan cuya tasa de mortalidad infantil es de 5.3 y las Islas Marianas del Norte, con una tasa de 5.5 por mil r.n.v. En Burkina Faso, Guinea y Malawi, las tasas de mortalidad infantil son de 137,143 y 149 por cada 1,000 nacimientos y la espera de vida no llega a los 50 años.

Por otro lado, la mortalidad materna en el mundo es aún muy elevada, con 390 defunciones por cada 100 mil nacimientos, pero con una diferencia de 30 en los países desarrollados y de 450 en los que aún están por debajo de los estándares del desarrollo. En los extremos se puede observar que la tasa de mortalidad materna en Suecia y Bélgica es menor de 5, en tanto que en Bangladesh y Etiopía es mayor de 500. 
Estos indicadores, muy generales, no permitirían observar una polarización epidemiológica de los países en el mundo. Con ello podría intentarse plantear asimismo, que los países situados en los intermedios de una lista referida, se encontrarían en el proceso de la transición epidemiológica, dado la disminución de muertes tempranas, la consecuente oportunidad de llegar a edades mayores y la mayor probabilidad de enfermar o morir por causas crónicodegenerativas. Así, el patrón de morbilidad y mortalidad se modifica mediante el traslape de enfermedades crónicas, no transmisibles y las infecciosas. Este fenómeno ocurre en diferentes tiempos y a velocidades distintas, por lo que el impacto no parece ser igual, de tal manera que resulta más evidente en la subregión de América Latina, que en África y los países más pobres de Asia.

Prueba de esta hipótesis es que en el siglo pasado casi todas las enfermedades ahora etiquetadas como tropicales, existieron también en la región templada de América Latina y Europa. No hay una explicación satisfactoria sobre la desaparición de la lepra en el norte de Europa, aunque se atribuye, en general, al mejoramiento socioeconómico $\mathrm{y}$, como consecuencia, de las condiciones de vida.

Fue así como el dengue acuñó su nombre durante la epidemia de Nueva Cork y Filadelfia, en la segunda mitad del siglo pasado; la tuberculosis, no respetaba el rango social de sus víctimas y, hasta la segunda guerra mundial, la malaria asolaba el sur de los Estados Unidos, el sur de Europa y el norte de África. En la actualidad, estas enfermedades las encontramos casi exclusivamente en el cinturón intertropical.

Se estima el número anual de casos de malaria entre los 250 y los 300 millones, en 103 países, donde vive cerca de la mitad de la población mundial total.

Los casos anuales de tuberculosis pulmonar bacilíferos, son de 4 a 5 millones y si se les suman los casos con baciloscopía negativa, la cifra puede rebasar los 10 millones anuales; el número de defunciones por esta enfermedad fluctúa alrededor de los 3 millones cada año. De los más de 5 millones de enfermos de lepra registrados en 1988, $75 \%$ son del sureste asiático, $12 \%$ de África, $7 \%$ de las Américas y el resto de otras regiones.

De acuerdo con Omram, el principal teórico de la transición epidemiológica, esta ocurre en tres etapas sucesivas a las que denomina: 1) la de las pestilencias y hambrunas, la Europa en el siglo pasado estaría en esta etapa y países como Somalia, Bangladesh y Etiopía en la actualidad; 2) la etapa del descenso de las pandemias, en donde se podrían ubicar la mayor parte de los países que se han identificado como en proceso de desarrollo o de ingresos medios, y 3) la etapa de las enfermedades degenerativas y producidas por el hombre, donde se ubicaron países como Japón, Estados Unidos, Canadá y Europa Occidental. El mismo autor identifica también tres modelos de transición epidemiológica: el clásico u occidental, que caracteriza a Europa, Norte América y Canadá; el acelerado, en el cual se identificarían Japón, Corea, Taiwan y otros países de la Cuenca del Pacífico Occidental y el contemporáneo o tardío, en el que se encuentran países de América Latina, Sri Lanka y otros países asiáticos.

El inicio de la transición epidemiológica coincide, y puede ser atribuido en gran parte, al desarrollo de tecnologías para la protección y atención a la salud. La velocidad con 
que el proceso transicional ocurre tiene que ver con la capacidad de la infraestructura de salud de los gobiernos para implantar, desarrollar y mantener o concluir, con éxito, programas de salud en la población y, también con el nivel de satisfactores sociales de que dispone la población, incluyendo sus niveles de educación. El fenómeno de la transición epidemiológica puede ser muy prolongado, en tanto que los recursos de la tecnología agoten por saturación las posibilidades de lograr nuevas transiciones, sobre todo en la mortalidad y la morbilidad. Algunos países, cuyas posibilidades de modificación estructural de la sociedad, o no ocurre o es muy lenta, se verán sumidos en una cuestionable transición, que dejaría de serlo por el alargamiento impredecible de un patrón epidemiológico mixto y escasamente cambiante y con pocas posibilidades de revolucionarse.

Frenk y Sepúlveda en México, se refieren a la transición epidemiológica como parte de la transición en salud, que incorpora además de la transición epidemiológica per se, aspectos como el envejecimiento de la población, el cambio de atención de la morbilidad y el predominio de los servicios ambulatatorios sobre los de hospitalización.

\section{LA TRANSFORMACIÓN DE LOS SISTEMAS NACIONALES DE SALUD.}

Acorde al contexto ya definido, en el que se precisan el estado de salud y su entorno socioeconómico y político, los países del orbe han emprendido la transformación de sus sistemas de Servicios de Salud, principal condicionante para el logro de la meta de Salud para Todos, con base en la estrategia de atención primaria, apoyándose en la descentralización y el fortalecimiento de los sistemas de salud a nivel local. En este proceso de transformación, la concepción sistémica e integral de los distintos componentes de la infraestructura, entendida en su sentido más amplio, adquieren un papel preponderante en la mejoría del impacto de las acciones emprendidas.

La evolución de los sistemas de salud, hacia servicios más equitativos, efectivos y eficientes, ha exigido modificaciones en la función, el financiamiento y con gran frecuencia, cambios estructurales significativos, especialmente en campos críticos como el estilo de conducción, especialmente si existe un sistema plural en el financiamiento y entrega de servicios, cuyos objetivos son compartidos por las partes; el sector público debe ejercer un liderazgo que oriente, estimule y regule el funcionamiento del Sistema y sus organismos de ejecución, incluidos los sectores social y privado.

La redefinición del papel del Estado, de lo cual ya algo se mencionó, trae como resultado implicancias en la reorganización del Sector Salud, en el mejoramiento de los servicios a las personas y al medio; además de que también dimensiona las relaciones entre los servicios de salud y las acciones de bienestar social, así como de las relaciones entre las instancias centrales de gobierno, las instancias públicas periféricas y las organizaciones de la sociedad civil.

Entre los principales propósitos de la reorganización del sector, está la de transformar los estilos gerenciales y la capacidad administrativa para procurar una gestión más efectiva y ágil, altamente productiva y de calidad aceptable. Simultáneamente, se requiere iniciar una intensa movilización de recursos, nacionales e internacionales, para 
lograr la transformación de los Sistemas de Salud de acuerdo a las necesidades de la población.

En la región de las Américas en la Actualidad, dentro de esta realidad se reafirma el liderazgo del Ministerio de Salud, como organismo rector del Sistema y de la política y acciones de salud, generalmente a través de 3 grandes áreas de actividad institucional; la prestación de servicios médicos, la protección y desarrollo de la salud pública comunitaria y el fortalecimiento de los llamados servicios sociales y de bienestar.

La legislación de salud, parte integrante de la infraestructura de los servicios de salud, constituye otro de los objetivos que se debe incrementar en la actualidad, especialmente en los países en desarrollo, en los que la legislación es débil e incompleta; se espera lograr en adelante, el desarrollo de un cuerpo de legislación moderna y efectiva, con sus reglamentos y normas que faciliten el funcionamiento adecuado del sistema.

Los avances en esta materia son considerables en el último lustro, tanto en su aspecto de derecho constitucional, como en el ámbito reglamentario.

El apoyo económico para el sostenimiento de los Sistemas de Salud y su funcionamiento, generalmente requiere fondos de distintas fuentes; fiscales de procedencia nacional, provincial y local; privados, en todas sus formas; de los seguros sociales y diversos tipos de financiamiento especial. En los sistemas de salud se encuentran combinaciones de los mismos y distintos mecanismos para su recaudación y manejo. En los países en desarrollo el financiamiento externo aún constituye en muchos casos un aporte considerable.

En la actualidad, conforme la restricción general del gasto público, la mayoría de los gobiernos ha reducido sustancialmente sus gastos en salud. Es frecuente comprobar que el gasto en Salud comienza a declinar, de los últimos años de los 70' hasta fines de los 80 ', aunque no es común a todos los países. Información reciente indica que a nivel mundial el $25 \%$ de los países tienen un gasto en salud de cerca de $10 \%$ del gasto total, mientras que otro $25 \%$ lo tiene por debajo del 3\%. En términos generales, de 1981 a 1983 hubo necesitados.

En julio de 1991 en Morelos, México, los Ministros de Salud de 11 países de distintos continentes discutieron respecto de la participación pública privada en el desarrollo de los sistemas de salud, y su inclusión en la determinación de metas del Sistema Nacional de Salud, el aprovechamiento al máximo de los recursos disponibles y el nuevo papel de los Ministerios de salud. En el desarrollo de esta reunión se concluyó que las políticas de la privatización deben ser evaluadas, teniendo en cuenta la eficacia, equidad y el costo de implementación. La regulación de la participación del sector privado, es un área que ha recibido un énfasis inadecuado y que convendría desarrollar estructuras de incentivos apropiados y vías para promover la competitividad y asegurar una calidad satisfactoria en ese sector.

De acuerdo a la experiencia, la participación del sector privado presenta aspectos conflictivos o complementarios, por lo que se recomendó una mayor investigación para 
conocer mejor el papel que debería jugar dentro del Sistema Nacional de Salud y la experimentación, con nuevos modelos, para su adecuado estudio.

Por otro lado, a pesar de las grandes restricciones financieras, los países han continuado sus esfuerzos para fortalecer la coordinación intersectorial. Dentro de este esfuerzo y como resultados apreciables se pueden mencionar los alcanzados en China, Costa Rica, Cuba y Sri Lanka, en donde se combinan los beneficios de un sistema sanitario eficaz, acompañado de mejoras en la agricultura, la educación básica y las políticas sociales en general.

Después de más de 12 años de haberse definido la meda de Salud para Todos en el año 2000, la descentralización política, administrativa y territorial sigue siendo un tema de constante preocupación para la mayoría de los países del mundo, que la definen junto con el desarrollo local, como la base para la reorganización del Sector, dentro de la estrategia de Atención Primaria. La descentralización, cuando se lleva a cabo en forma adecuada, permite que a nivel local se responda a las necesidades individuales, familiares y comunitarias, de manera eficaz y oportuna.

En la actualidad el $65 \%$ de los países de la región de las Américas están descentralizando la administración pública, en medio de la crisis económica y como parte de su intento para mejorar la accesibilidad, la eficiencia y la eficacia de los servicios sociales en general y de los de salud en particular.

En la región de las Américas, se ha definido al desarrollo de los Sistemas Locales de Salud (SILOS) como una táctica operativa para acelerar la aplicación de la estrategia de Atención Primaria.

En este tenor, la participación social, como compromiso pleno, consciente y activo, presenta diferencias sustanciales de interpretación y operatividad en los distintos países y continentes; en ese sentido, las dimensiones de la participación varían en relación a los mecanismos y modalidades, la amplitud de la misma y de los distintos campos en que se aplica.

Como complemento necesario, la situación de los recursos humanos para la salud a nivel mundial, se considera un área crítica. La característica sobresaliente es una deficiencia profunda y generalizada, con profesionales poco adiestrados y sobre todo sin motivación; frecuentemente la contratación y el adistramiento están completamente separados y muestran grandes incoherencias; la principal causa de esta problemática pareciera estar en la práctica de la gestión y en los sistemas de apoyo del personal.

En cuanto a su capacidad generadora de empleo, aunque el sector salud tiene apreciable importancia, en la actualidad en la mayoría de países en desarrollo y en algunos países desarrollados, la posible disminución de la masa salarial como resultado de la crisis, puede generar múltiples problemas para el mantenimiento de la fuerza de trabajo. En aspectos de organización existe un amplio margen de modalidades de inserción y de los montos salariales que determinan e impactan el trabajo en salud, desde la incorporación al régimen de contrato asalariado a tiempo fijo, hasta la práctica liberal pura. 
En lo que toca a los recursos físicos, la aceptación por parte de la mayoría de países de la política de descentralización y el fortalecimiento de los Sistemas Locales, hace necesaria la revisión de los indicadores de la capacidad instalada y de la oferta de los servicios de salud y sus grados de utilización, a fin de crear verdaderas redes de servicios, integradas y autosuficientes, con adecuados mecanismos de referencia, lo cual se interrelaciona estrechamente con la necesidad de desarrollar nuevos modelos de atención.

El mantenimiento y la operación adecuada y permanente del equipo existente, no se ha concretado en una prioridad del mundo en desarrollo, en general. Como resultado, se manifiestan graves deficiencias en ese campo; hay falta de políticas expresas de mantenimiento y conservación; poca capacidad institucional; falta de subsistemas nacionales de ingeniería y mantenimiento, y falta de programas de mantenimiento preventivo. Los recursos económicos en América Latina destinados a mantenimiento oscilan entre $0.6 \%$ y $3.4 \%$ de los gastos de operación, lo que nos sitúa muy por debajo de los porcentajes técnicamente recomendados.

Como resultado de estas múltiples deficiencias, entre el 35\% y 53\% de los equipos están paralizados, el promedio de los equipos tienen 10 años y existe una brecha entre el avance tecnológico del equipo y la capacidad técnica del personal que lo maneja.

Así mismo, en la actualidad son pocos los países con redes de laboratorios bien desarrollados. La complejidad y alto costo de los procedimientos diagnosticados y terapéuticos, está creando preocupación en los países en desarrollo así como en los desarrollados; la transferencia de tecnología ha sido hecha en forma indiscriminada y muchas veces sin considerar a fondo sus ventajas e inconvenientes. En el plano ético, la alta tecnología ha acaparado recursos que harían falta para la atención de problemas prioritarios a tratar con tecnologías poca complejas.

Finalmente, la producción y el suministro, la calidad y el adecuado uso de los medicamentos, de acuerdo a la estrategia de Atención Primaria de Salud, constituyen áreas prioritarias para el mejoramiento y ampliación de cobertura de los servicios de salud. Durante el decenio de 1980, los países han realizado importantes logros, ejemplificados por los esfuerzos para racionalizar la prescripción en el uso de los medicamentos, especialmente a través de la elaboración (o actualización) de los cuadros básicos de medicamentos esenciales y sus respectivos formularios terapéuticos.

Sin embargo, los progresos alcanzados no han sido suficientes para responder a las necesidades, y grandes sectores de la población no tienen acceso a los medicamentos básicos; las áreas críticas del suministro siguen siendo un proceso de compras inoperante, con almacenamiento inadecuado, deficiente distribución y uso irracional de los mismos. Además no existe en la mayoría de países un sistema de datos confiables, ni instrumentos de evaluación de programas y servicios, en términos de eficacia, eficiencia y calidad.

Respecto al gasto de medicamentos, las cifras correspondientes a la América Latina, nos muestran un descenso sostenido en los últimos 6 años (1980-1986), que va desde - 
0.2 en Perú a -24.4 en Argentina, en contraste con +30.5 en E.E.U.U y +15.7 en Canadá; Ecuador constituye la única excepción con +0.7 .

Por su parte el mercado farmacéutico constituyó en 1980 el 6.5\% del mercado mundial, y en 1987 únicamente el 3.6 con una reducción de $2.9 \%$. La producción de biológicos en América, por subregiones, muestra diferentes niveles de producción; Canadá, E.E.U.U. y México producen todas las vacunas del PAI; los dos primeros con autosuficiencia y México en un 60\%. América Central y el Caribe, con excepción de Cuba, no tiene capacidad de producción y América del Sur en conjunto sólo satisface menos del 50\% de sus necesidades.

\section{APRECIACIÓN GENERAL}

La salud pública ha creado su propio espacio, el mismo que no está mereciendo la prioridad y atención adecuadas. Su práctica actual contempla lo que se puede hacer en medicina y atención médica, no obstante no nos limita ni subordina exclusivamente a ellas. Estamos viviendo la época de las transiciones, primero poblacional, luego epidemiológico, seguida de la transición en salud y ahora creo que debemos considerar la transición programática.

Se ha migrado de la preocupación exclusiva por la enfermedad, hacia la atención a la salud, no solo en lo convencional sino incluyendo los estilos de vida saludables y asociando la salud con el bienestar. Es parte, en consecuencia, del trabajo en salud pública el preocuparse por el desarrollo necesario.

La dimensión social de la salud le da a la política, en su sentido más amplio, que es el de la organización, estructura y ejercicio del poder en la sociedad, quizás el punto más relevante para el proceso de cambios que debe promover.

Se contraponen posiciones entre los que toman las decisiones y lo que es necesario hacer, la continuidad versus el cambio, las demandas versus las necesidades y la acción inmediata-que por lo general es coyuntural-con aquellas que tienen como objetivo el futuro. Todo esto requiere su compatibilización, el balance entre focalización, verticalidad, especialización y generalidad e integración.

Finalmente dentro del contexto referido, mi mensaje es de confianza, en lo que los hombres y mujeres, con la libertad de iniciativa y la capacidad de creación individual o en conjunto, en este mundo fascinante que vivimos, contribuirán a enfrentar los desafíos para la salud pública de nuestros tiempos.

\section{BIBLIOGRAFÍA}

1.Banco Interamericano de Desarrollo. América Latina. Notas sobre el Decenio de 1990. Primera Cumbre Iberoamericana. Guadalajara, Jal. México, 1991.

2.Banco Mundial. Informe sobre el Desarrollo Mundial, 1991. La Tarea Acuciante del Desarrollo. Washington, D.C. 1991. Oxford University Press. 
3.Carrasco, L.R., Gonzáles T.E., Provencio, E. La cuestión del Desarrollo en América Latina. Problemas, Referencias y Lineamientos. Investigación Económica 194, octubrediciembre de 1990, pp.139-184.

4.Comisión Económica para América Latina y el Caribe. Notas sobre el Desarrollo Social en América Latina. Primera Iberoaméricana. Guadalajara, Jal. México, 1991.

5.Fondo de Población de las Naciones Unidas. Estado de la Población Mundial 1991. Nueva Cork, N.Y. 1991.

6.Frenk, J. y cols. Elementos para una Teoría de la Transición en Salud. Salud Pública de México. (33) 5, 348-462.

7.Organización de Estados Americanos. Informe del Grupo Ad hoc Encargado del Estudio de la Iniciativa para las Américas. Washington, D.C, 1991.

8.Organización Mundial de la Salud. De Alma-ata al año 2000. Reflexiones a Medio Camino. Ginebra, 1991.

9. Organización Mundial de la Salud. Fortalecimiento de los Ministerios de Salud para el Fomento de la Atención Primaria. Ginebra, 1990. Serie Informes Técnicos 766.

10.Organización Panamericana de la Salud/ Organización Mundial de la Salud. Las Condiciones de Salud en las Américas Washington, D.C., 1990, Publicación Científica N. 524.

11.Organización Panamericana de la Salud/ Organización Mundial de la Salud. La Salud en el Desarrollo. Comité Ejecutivo del Consejo Directivo, Decimoquinta Reunión. Washington, D.C, 6 de diciembre de 1990.

12. Organización Panamericana de la Salud/Organización Mundial de la Salud. La Salud como Pilar Fundamental del Desarrollo Social de Iberoamerica. Primera Cumbre Iberoamericana. Guadalajara, Jal. México, 1991.

13.Organización Panamericana de la Salud/ Organización Mundial de la Salud. Los Sistemas de Salud. Desarrollo y Fortalecimiento. Washington, D.C., 1989.

14. Organización Panamericana de la Salud/Organización Mundial de la Salud. Los Sistemas Locales de Salud. Washington, D.C., 1990, Publicación Científica $\mathrm{N}^{\circ} 519$

15. Organización Panamericana de la Salud/ Organización Mundial de la Salud. La Salud como Pilar Fundamental del Desarrollo Social de Iberoamérica. Primera Cumbre Iberoamericana. Guadalajara Jal. México, 1991

16.Organización Panamericana de la Salud/Organización Mundial de la Salud. Sistemas Locales de Salud, Informe de Progreso. Washington, D.C., 1990 HSD/SILOS, N 10 17.Paganini, J.M. Los desafíos de los Servicios de Salud, en la Década de los 90's en los SILOS de América Latina. Caracas. Organización Panamericana de la Salud. 1990

18.Paiwe, L.H.W. y Siem Team, F. Los Hospitales y la Revolución de la Asistencia Sanitaria. Ginebra, Organización Mundial de la Salud, 1989

19.Programa de Naciones Unidas para el Desarrollo. Desarrollo Humano: Informe 1991. Bogotá, Colombia 1991. Tercer Mundo Editores.

20.Rennet, Sara-Mills, Anne. The Public/Private Mix in Nacional Health Systems. Iteregional Meeting. Cocoyoc, Mor. México, Organización Mundial de la Salud, 1991 21.Tabibzadeh A. y Espasnet A. Ross. La Ciudad en Primer Plano. Ginebra, Organización Mundial de la Salud, Ginebra, 1990. 